OPEN ACCESS

Edited by:

Alejandra A. Covarrubias, National Autonomous University of Mexico, Mexico

Reviewed by: Kazuo Nakashima, Japan International Research Center for Agricultural Sciences, Japan Yong Hwa Cheong, Sunchon National University, South Korea

*Correspondence: Shouping Yang spyung@126.com

Specialty section: This article was submitted to Plant Abiotic Stress,

a section of the journal Frontiers in Plant Science

Received: 17 August 2019 Accepted: 05 December 2019 Published: 29 January 2020

Citation:

Li C, Liu X, Ruan H, Zhang J, Xie F, Gai J and Yang $S$ (2020) GmWRKY45 Enhances Tolerance to Phosphate Starvation and Salt Stress, and Changes Fertility in Transgenic Arabidopsis.

Front. Plant Sci. 10:1714. doi: 10.3389/fp/s.2019.01714

\section{GmWRKY45 Enhances Tolerance to Phosphate Starvation and Salt Stress, and Changes Fertility in Transgenic Arabidopsis}

\author{
Cheng Li, Xinyi Liu, Hui Ruan, Jingyao Zhang, Fengbin Xie, Junyi Gai and Shouping Yang* \\ Soybean Research Institute, National Center for Soybean Improvement, Key Laboratory of Biology and Genetic Improvement of \\ Soybean (General, Ministry of Agriculture), State Key Laboratory of Crop Genetics and Germplasm Enhancement, Jiangsu \\ Collaborative Innovation Center for Modern Crop Production, Nanjing Agricultural University, Nanjing, China
}

WRKY protein is a unique transcription factor (TF) and plays an important role in the physiological processes of various stress responses and plant development. In this research, we obtained a WRKY TF gene from soybean by homologous cloning, and named it GmWRKY45. GmWRKY45 is a nuclear protein containing a highly conserved WRKY domain and $\mathrm{a}_{2} \mathrm{H}_{2}$ zinc finger structure, and mainly expressed in roots, flowers and pods of soybean. The quantitative reverse transcription-PCR showed that GmWRKY45 was induced by phosphate starvation and salt stress. As compared with the wild type (WT), overexpression of GmWRKY45 increased the adaptability of transgenic Arabidopsis to phosphate starvation, which might be related to the enhancement of lateral root development. The phosphorus concentration, fresh weight and dry weight of GmWRKY45-overexpressing Arabidopsis were higher than those of WT under Pi-sufficient or Pi-deficient condition. Meantime, the expression of phosphateresponsive genes was affected in transgenic Arabidopsis. Furthermore, GmWRKY45 improved the salt tolerance and changed fertility of transgenic Arabidopsis. Under salt stress, we found the survival rate and soluble sugar content of transgenic Arabidopsis were significantly higher than those of WT. In a conventional soil pot experiment, the transgenic Arabidopsis produced shorter silique, less and larger seeds than WT, these might be due to partial abortion of pollens. The overall results showed that GmWRKY45 was not only involved in response to abiotic stress but also related to fertility, suggested that GmWRKY45 had an elaborate regulatory system in plants.

Keywords: GmWRKY45, soybean (Glycine max), transgenic Arabidopsis, tolerance, phosphate starvation, salt stress, fertility

\section{INTRODUCTION}

In the natural environment, plants will encounter various biological and non-biological pressures during their growth and development. Phosphorus $(\mathrm{P})$ is one of 17 essential elements required for plant growth. P plays an important role in plant growth and development, including energy generation, nucleic acid synthesis, photosynthesis, glycolysis, respiration, membrane synthesis and 
stability, etc. Although $\mathrm{P}$ is abundant in many soils, crop yield on $30 \%-40 \%$ of the world's arable land is limited by $\mathrm{P}$ availability, because plants take up $\mathrm{P}$ exclusively in the form of inorganic phosphate (Pi) (Vance et al., 2003). Soil salinity is also a major abiotic stress worldwide (Rengasamy, 2006). In irrigated land areas, continuous salinization of agricultural land is a serious threat to crop yields (Munns and Tester, 2008). Therefore, the development of plants that can efficiently resist phosphate starvation and salt stress are a sustainable and economic approach for plant production, thus it may be an important contribution for the improvement of crop yield.

In recent years, scientists have discovered several key molecules involved in $\mathrm{Pi}$ signal transduction and salinity tolerance, further improving the understanding to plant phosphorus utilization and salt tolerance at the molecular level (Zhang et al., 2016; Xu et al., 2018). By using genetic methods, many families of transcription factors have been found to play important roles in plant phosphorus signaling and salt tolerance, including WRKY, AP2/ERF, MYB, bHLH, and NAC family members (Baek et al., 2013; Phukan et al., 2016). These TFs usually bind to specific cis-acting elements in the promoter region and regulate the expression of several stress response genes. For example, AP2/ERF can be combined with GCC box and DRE/CRT components and induced the expression of some PR genes, thus improving the high salt tolerance of plants (Zhang et al., 2009).

The WRKY TF superfamily is one of the largest TF families and almost all are found in plants. The WRKY transcriptional factor superfamily has 72 members in Arabidopsis (Arabidopsis thaliana) and 182 members in soybean (Glycine max), and its definition is based on the highly conserved WRKY domain (Eulgem and Somssich, 2007; Bencke-Malato et al., 2014). The WRKY domain is a conserved DNA-binding region that includes highly conserved WRKYGQK peptide sequences and zinc finger motifs which can be either $\mathrm{C}_{2} \mathrm{H}_{2}$-type $\left(\mathrm{Cx}_{4-5} \mathrm{Cx}_{22-23} \mathrm{HxH}\right)$ or $\mathrm{C}_{2} \mathrm{HC}$-type $\left(\mathrm{Cx}_{7} \mathrm{Cx}_{23} \mathrm{H}_{\mathrm{x}} \mathrm{C}\right)$. Members of the WRKY family have been found to contain at least one such domain. This domain generally binds to the promoter region of target genes containing the $\mathrm{W}$-box sequence (C/TTGACT/C), although alternative binding sites have been identified (Pandey and Somssich, 2009). W-box elements are prevalent in plant genomes. For example, 32,162 TTGACY, 60,612 TTGAC, and 14,857 TTTGACY were identified in Arabidopsis (Chen et al., 2017). The current and widely accepted system of WRKY classification was established in 2000 based on the genomic characterization of this gene family in Arabidopsis (Eulgem et al., 2000). All WRKY TFs are classified into three categories: I, II, and III, based on the number of WRKY domains and the type of zinc finger figure. Group I WRKY proteins harbor two WRKY domains and a $\mathrm{C}_{2} \mathrm{H}_{2}$-type zinc-finger structure, whereas groups II and III WRKY proteins contain only one WRKY domain with $\mathrm{C}_{2} \mathrm{H}_{2}$ and $\mathrm{C}_{2} \mathrm{HC}$ type zinc-finger structures, respectively. Group II proteins are further categorized into five subgroups (IIa-IIe) (Rushton et al., 2010). WRKY transcription factors have been shown to be involved in responses to biotic and abiotic stresses, and in developmental processes (Eulgem and Somssich, 2007).
Transgenic Arabidopsis plants over-expressing GmWRKY13 showed increased sensitivity to salt and mannitol stresses, and decreased sensitivity to abscisic acid (ABA), when compared with wild-type plants (Zhou et al., 2008). GmWRKY21-transgenic Arabidopsis plants were tolerant to cold stress (Zhou et al., 2008). GmWRKY54-transgenic Arabidopsis plants conferred salt and drought tolerance (Zhou et al., 2008). According to the newest publication, AtWRKY45 overexpression in Arabidopsis increased Pi content and uptake, while RNA interference suppression of AtWRKY45 decreased Pi content and uptake (Wang et al., 2014). Since Arabidopsis is a dicotyledonous model plant, we speculate that GmWRKY45 in soybean may have a similar function. However, WRKY protein with structural homology in different plants may have different functions, which needs experimental verification (Yang et al., 2016).

Soybean is one of the most important crops for oil and protein production, and its yield is severely affected by various environmental conditions (Keaton et al., 2013). In this research, we cloned the full-length cDNA of GmWRKY45, and report its expression under phosphate starvation and salt stress. We found that overexpression of GmWRKY45 increased tolerance to phosphate starvation and salt stress in transgenic Arabidopsis. Interesting, we also found the transgenic Arabidopsis produced shorter silique, less and larger seeds. These results showed that GmWRKY45 was not only involved in response to abiotic stress, but also related to fertility, suggesting that GmWRKY45 had an elaborate regulatory system in plants.

\section{MATERIALS AND METHODS}

\section{Phylogenetic and Gene Structure Analysis}

Using AtWRKY45 cDNA sequence of Arabidopsis as probe, BLAST retrieval was conducted using the National Center of Biotechnology Information (www.ncbi.nlm.nih.gov/), find the target gene. BioXM 2.6 software was used to predict the molecular weight and isoelectric point of the gene, and GSDS (http://gsds.cbi.pku.edu.cn/) online program was used to predict the gene structure. The conserved functional domains of the gene were predicted by using SMART (http://smart.embl-heidelberg. $\mathrm{de} /$ ) and NCBI conserved structural domain database (CDD) (https://www.ncbi.nlm.nih.gov/Structure/cdd/wrpsb.cgi). ClustalX 1.83 and GeneDoc were used for multiple alignments. Neighbor-joining phylogenetic trees were generated using the MEGA 5.1 program.

\section{Plant Material, Growth Conditions, and Stress Treatments}

Seeds of soybean genotype Williams 82 were used to investigate the tissue expression pattern of GmWRKY45 and its responses to various stresses including phosphate starvation and salt stress. Phosphate starvation: Soybean seeds germinated in vermiculite medium for five days were transferred to Hoagland solution and treated with two Pi levels (low $\mathrm{Pi}, 2.5 \mu \mathrm{M} \mathrm{KH}_{2} \mathrm{PO}_{4}$; normal $\mathrm{Pi}, 1$ $\mathrm{mM} \mathrm{KH_{2 }} \mathrm{PO}_{4}$ ) for 10 days (removal of cotyledons). A low $\mathrm{Pi}$ group re-supplied with Pi for 1 day was called Recovery 11d. Salt 
stress: Soybean seeds germinated in vermiculite medium for five days were transferred to Hoagland solution and treated with two $\mathrm{NaCl}$ levels ( $0 \mathrm{mM} \mathrm{NaCl} ; 150 \mathrm{mM} \mathrm{NaCl}$ ) for five days (removal of cotyledons). A $150 \mathrm{mM}$ level $\mathrm{NaCl}$ group was transferred to 0 $\mathrm{mM} \mathrm{NaCl}$ medium for 1 day, called Recovery 6d. Leaves and roots were sampled after transfer. All samples were frozen in liquid nitrogen and stored at $-80^{\circ} \mathrm{C}$ prior to RNA extraction.

\section{Quantitative Real-Time PCR (RT-qPCR)}

Three independent biological replicates, each comprising three individual plant, were used for quantitative real-time PCR. The genomic DNA was removed and the total RNA was converted to cDNA using the HiScript II 1st Strand cDNA Synthesis Kit (Vazyme Biotech, Nanjing, China). Quantitative real-time PCR was performed on a BioRad CFX96 real time system and the products were labeled using SYBR qPCR Master Mix (Vazyme Biotech, Nanjing, China). Triplicate quantitative assays were performed on each cDNA sample. All primers used for RTqPCR are listed in Supplemental Tables S1 and S2.

\section{Arabidopsis Protoplast Isolation and Subcellular Localization}

The coding sequence (CDS) without a termination codon of GmWRKY45 (861 bp) was used to construct the pJIT166GmWRKY45-GFP vector driven by the CaMV35S promoter. Arabidopsis protoplasts were isolated by following the previously published protocol (Yoo et al., 2007). Both fusion (pJIT166-GmWRKY45-GFP) and control ( $p J I T 166-G F P$ ) constructs were transformed into Arabidopsis protoplast by the PEG4000-mediated method as described by Abel and Theologis (1994). The GFP fluorescence was imaged using a Zeiss LSM780 camera (Carl Zeiss, SAS, Jena, Germany).

\section{Development of Transgenic Arabidopsis}

To generate GmWRKY45-overexpressing transgenic Arabidopsis lines, full-length cDNA of the GmWRKY45 gene from Williams 82 was amplified. An over-expression construct was generated by inserting a full-length GmWRKY45 cDNA fragment into the binary vector $p C A M B I A 3301-G F P$ after the CaMV35S promoter, using the Gateway Technology with a One Step Cloning Kit (Vazyme Biotech, Nanjing, China) according to the manufacturer's protocol. The sequences of the primers are provided with Supplemental Table S3.

In this study, Arabidopsis ecotype Columbia-0 (Col-0) was used. The pCAMBIA3301-GmWRKY45 vector was introduced into Agrobacterium tumefaciens strain EHA105 via the freeze-thaw method. The Agrobacterium-mediated floral dip method was used for Arabidopsis transformation (Clough and Bent, 1998). Transgenic plants were selected on Basta $\left(20 \mathrm{mgL}^{-1}\right)$ medium, and the selected plants were identified by PCR (Supplemental Figure S1). Homozygous T3 or T4 seeds were used for researching.

\section{Measurement of Chlorophyll and Anthocyanin Contents}

Two-week-old plants were grown in Hoagland medium with two Pi levels $\left(\mathrm{P}+, 1 \mathrm{mM} \mathrm{KH}_{2} \mathrm{PO}_{4}\right.$; $\left.\mathrm{P}-, 0.25 \mathrm{mM} \mathrm{KH}_{2} \mathrm{PO}_{4}\right)$ for 30 days. The chlorophyll content was assayed by SPAD-502 Plus (Japan).
The anthocyanin content was assayed referred to Kim et al. (2003) and a little modification: Take plant leaf blade $0.01 \mathrm{~g}$, in 300 ul $1 \% \mathrm{HCl}$-methanol mixture, $4^{\circ} \mathrm{C}$ temperature overnight,

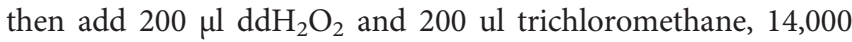
rpm centrifuge for $15 \mathrm{~min}$ and drain the upper water phase into the enzyme label plate, enzyme standard instrument for determination of $530 \mathrm{~nm}$ and $657 \mathrm{~nm}$ absorbance value. Anthocyanin formula: $\mathrm{A}_{530} 0.33 \mathrm{~A}_{657}$. Three plant samples were randomly selected from each treatment, and each experiment contained three biological replicates.

\section{Measurements of Lateral Root Length and P Concentration}

Seedlings were grown on square petri dishes under $\mathrm{P}+(1 \mathrm{mM}$ $\left.\mathrm{KH}_{2} \mathrm{PO}_{4}\right)$ and $\mathrm{P}$ - $\left(0.25 \mathrm{mM} \mathrm{KH_{2 }} \mathrm{PO}_{4}\right)$ milieu for 15 days. The roots were photographed under the scanner (Epson, Expression 11000XL, Japan) The length of lateral roots in individual plants was measured using the ImageJ program (Abràmoff et al., 2004). Data were recorded from five individual plants from each line per treatment. Inorganic phosphorus and total phosphorus concentration were determined using Tissue Total Phosphorus Content Detection Kit (Solarbio, Beijing, China) and Tissue Inorganic Phosphorus Content Detection Kit (Solarbio, Beijing, China), respectively. Each experiment contained three biological replicates.

\section{Measurement of Survival Rate and Soluble Sugar Content}

For survival assay, surface sterilization was carried out on 24 seeds of GmWRKY45-overexpressing transgenic Arabidopsis and WT, and they were placed in Petri plates containing Murashige and Skoog (MS) basal medium supplemented with 0 and 150 $\mathrm{mM} \mathrm{NaCl}$, respectively. The petri dishes were placed in a growth chamber at $25^{\circ} \mathrm{C}$ for 7 days under $16 \mathrm{~h}$ photoperiod. The survival rate was recorded as the ratio of plants with true leaf normal greening after 7 days to the total number sown.

Two-week-old plants were transferred MS nutrient solution supplemented or not with $150 \mathrm{mM} \mathrm{NaCl}$ and grown for 5 days. Soluble sugar contents were referring to a method of Xiang et al. (2007). $0.01 \mathrm{~g}$ Arabidopsis fresh leaf was cut into debris and put into a clean tube; $1 \mathrm{ml}$ of $\mathrm{ddH}_{2} \mathrm{O}$ was added and then the tube was placed in a boiling water bath for $30 \mathrm{~min}$ to extract. After cooling to room temperature, $0.2 \mathrm{ml}$ extracts were taken and placed in another clean tube, added $1 \mathrm{ml}$ of $5 \%$ phenol and $5 \mathrm{ml}$ of concentrated sulphuric acid. The mixture was then well shaken. After standing for $30 \mathrm{~min}$, the aqueous extract was assayed for soluble sugar content at the wavelength of $485 \mathrm{~nm}$. Each experiment contained three biological replicates.

\section{Alexander Staining of Pollen and Measurement of Seed Size}

Alexander stain is a kind of plant pollen dye commonly used for nuclear staining. Plant pollen using this stain will turn purplish red. Take fresh flowers, carefully remove the petals and pistil, put the pollen on a glass slide, and add 2-3 drops of Alexander Stain, mix them thoroughly, immediately cover the glass slide, remove the excess liquid and observe under the microscope. 
The seed size was photographed under an OLYMPUS CX31 stereo microscope with a SPOT-RT digital camera attached to a computer (Olympus, Melville, NY, USA). The length and width of the seed was measured using the ImageJ program (Abràmoff et al., 2004). Data were recorded from three individual plants with 10 seeds used from each line.

\section{Promoter Cloning and GUS Assay}

To study the promoter activity, a 2-kb genomic region upstream of the translation initiation codon of GmWRKY45 gene was cloned into pCAMBIA3301-GUS at BamHI and XbaI sites (primers see Supplemental Table S4). The sequence correctness of the target promoter on the fusion construct was verified by sequencing. The fusion construct was introduced into Agrobacterium tumefaciens strain EHA105 by electroporation. The Agrobacterium-mediated floral dip method was used for Arabidopsis (Col-0) transformation (Clough and Bent, 1998).

GUS assay procedures were the same as described by Scarpeci et al. (2008).

\section{Statistical Analyses}

Statistical analyses were performed using Microsoft Excel 2003 and The SAS System for Windows V8. Significant differences were evaluated using the two-tailed Student's $t$-test or one-way ANOVA and Duncan's test. All test differences at $\mathrm{P} \leq 0.05$ were considered to be significant. All the error bars were SD (Standard Deviation) value.

\section{RESULTS}

\section{Structure, Homology Characterization, and Subcellular Localization of GmWRKY45}

According to the coding sequence (CDS) of AtWRKY45 (AT3G01970) gene in Arabidopsis thaliana, through NCBI database and Phytozome 11.0 database, homologous gene comparison was conducted to find the gene sequence with the highest homology with AtWRKY45 gene in soybean, which I'll call GmWRKY45 (Glyma.03G220800). GmWRKY45 was located on chromosome 3 of soybean, position Gm3 (42405222 42407091). Sequence analysis showed that it contained 3 exons, an 864 bp open reading frame encoding a polypeptide of 288 amino acids with predicted molecular mass of $32.37 \mathrm{kDa}$ (pI 6.95). The amino acid sequence encoded by the gene was compared analysis on NCBI website, and it was found that the protein encoded by GmWRKY45 had a conservative WRKY domain sequence. The amino acid sequence of GmWRKY45 (NP_001237422) was used as the probe, and the WRKY45 protein in Arabidopsis, Oryza sativa, Vitis amurensis, Brassica napus and Solanum lycopersicum was obtained by NCBI BLASTp tool: AtWRKY45 (NP_186846), OsWRKY45 (AAW63720), VaWRKY45 (AFK27601), BnaWRKY45 (ACH99806) and SlWRKY45 (NP_001304843). Multiple comparisons showed that the amino acid sequence of GmWRKY45 was relatively conservative, and the N-terminal of GmWRKY45 protein contained a WRKYGQK domain and a $\mathrm{C}-\mathrm{X}_{4}-\mathrm{C}-\mathrm{X}_{23}-\mathrm{H}-\mathrm{X}_{1}-\mathrm{H}$ zinc finger structure (Figure 1A), so GmWRKY45 belonged to the IIc subfamily in the WRKY transcription factor family (Chen et al., 2017). Phylogenetic trees constructed from amino acid sequences showed high homology between GmWRKY45 and AtWRKY45 (Figure 1B), so we speculated that they may have similar functions.

Next, we predicted the subcellular localization of GmWRKY45 protein through the website (http://www.csbio. sjtu.edu.cn/bioinf/Cell-PLoc-2/), (Chou and Shen, 2008), and the predicted results showed that it was located in the nucleus. To further verify the subcellular localization of GmWRKY45, the full-length GmWRKY45 cDNA without the termination codon was fused in-frame to the 5' end of GFP gene of the pJIT166 vector to obtain the pJIT166-GmWRKY45 fusion construct. By following the PEG-4000-mediated method, the fusion plasmid of PJIT166-GmWRKY45 and the PJIT166 vector as control were introduced into Arabidopsis protoplasts. The cells were observed by confocal laser scanning microscope. The fluorescence of GmWRKY45-GFP accumulated mainly in the nucleus, while the fluorescence of the control was distributed evenly throughout all parts of the cell including the nucleus and cytoplasm (Figure 1C). These results indicate that GmWRKY45 likely functions in the nucleus and has the general characteristics of transcription factors.

\section{Expression Patterns of GmWRKY45}

To clarify the abundance of GmWRKY45 transcripts in specific tissues, the total RNA were extracted from roots, stems, leaves, flowers, pods and seeds of soybean plants at first trifoliate (V1), full bloom (R2), and full seed (R6) stages, respectively. RT-qPCR analysis showed that GmWRKY45 was expressed in all tissues examined and relative abundance of GmWRKY45 transcripts in roots tissues (R2 and R6), flowers (R2) and pods (R6) (Figure 2A). In addition, we found that GmWRKY45 transcripts in roots have an increasing response with growth advance (V1 to R6). The expression pattern of the leaves changed significantly, with lowest transcription observed at the V1, highest at the R2, and intermediate at the R6 stages. In stem tissues of R2 and R6 stages, the expression of GmWRKY45 did not change significantly and was relatively stable.

The expression patterns of GmWRKY45 under low $\mathrm{Pi}(2.5 \mu \mathrm{M}$ $\mathrm{KH}_{2} \mathrm{PO}_{4}$ ) condition were evaluated by RT-qPCR using RNA samples extracted from leaves and roots, and normal Pi (1 mM $\mathrm{KH}_{2} \mathrm{PO}_{4}$ ) condition was used as control. The expression level of GmWRKY45 increased significantly with time under low Pi treatment (Figure 2B). Specifically, the increases of GmWRKY45 transcripts in the leaves and roots peaked at the 5 th day and 10th day of Pi starvation respectively, and decreased rapidly after recovery with $\mathrm{Pi}$ for 1 day (11d/Recovery). In addition, we found that the intensity of GmWRKY45 induced by low phosphorus in roots was higher than that in leaves (Figure 2B). This may imply that the response in GmWRKY45 to low phosphorus is more sensitive in the root. To determine whether the up-regulation of GmWRKY45 was specific to phosphate starvation, the responsiveness of GmWRKY45 expression to salt stress was also investigated and we used 
A

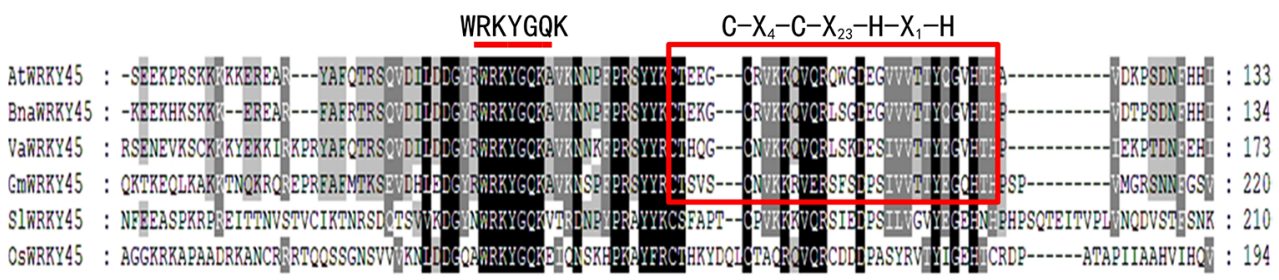

B
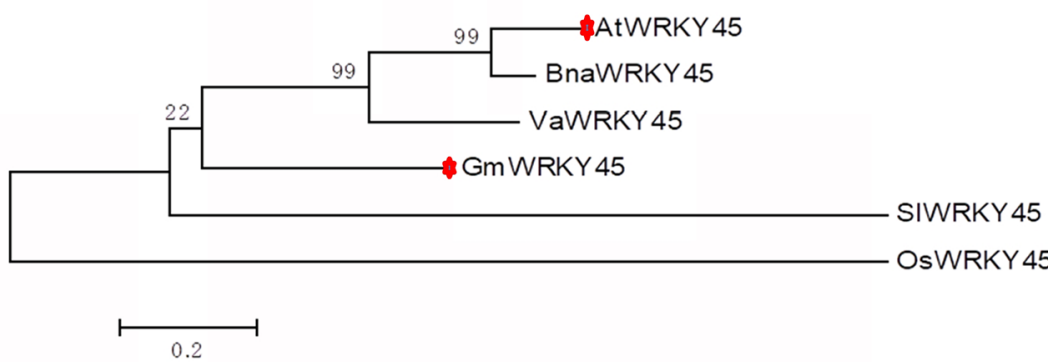

C
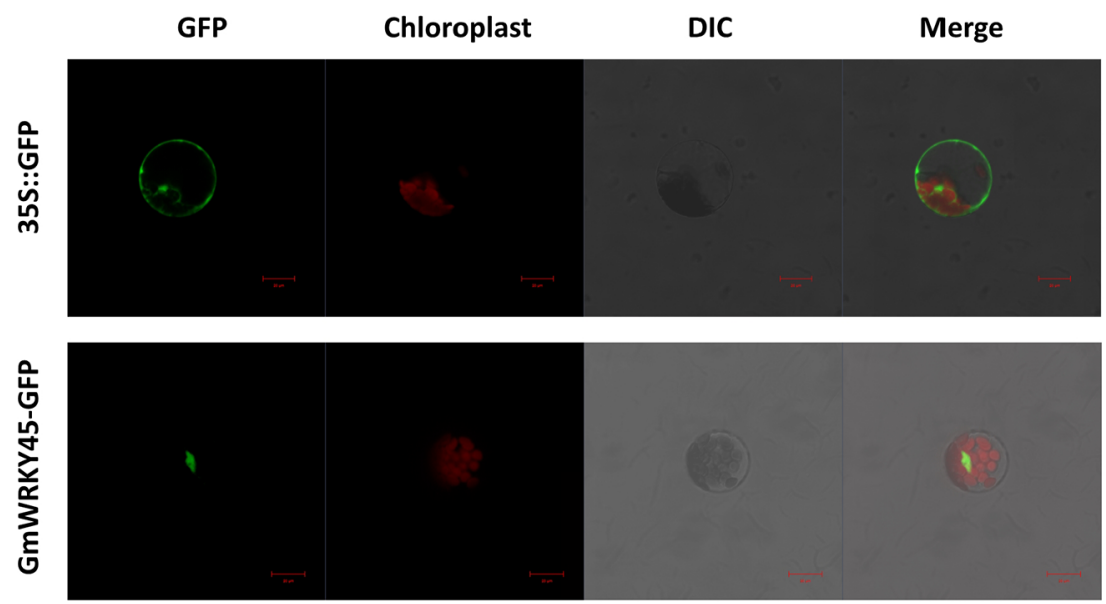

FIGURE 1 | Structure, homology characterization and subcellular localization of GmWRKY45. (A) GmWRKY45 and the WRKY45 protein from Arabidopsis (AtWRKY45), Oryza sativa (OsWRKY45), Vitis amurensis (VaWRKY45), Brassica napus (BnaWRKY45), and Solanum lycopersicum (SIWRKY45) were compared and analyzed by ClustalX 1.83 software. The same amino acids in all six proteins are shown in dark gray. The red bar above the sequences represents the highly conserved WRKYGQK domain. Red square represents the N-terminal C- $\mathrm{X}_{4}-\mathrm{C}-\mathrm{X}_{23}-\mathrm{H}-\mathrm{X}_{1}-\mathrm{H}$ zinc finger structure. (B) Based on the amino acid sequence of the selected GmWRKY45 protein, a phylogenetic comparison was made between the sequences of the GmWRKY45 protein and the WRKY45-related protein. The unrooted tree was constructed using MEGA 5.1 by the neighbor-joining method with bootstrap probabilities based on 1000 replicates shown at branch nodes. Accession numbers for the WRKY45 proteins used are as follows: GmWRKY45, NP_001237422; AtWRKY45, NP_186846; OsWRKY45, AAW63720; VaWRKY45, AFK27601; BnaWRKY45, ACH99806, and SIWRKY45, NP_001304843. (C) Subcellular localization of GmWRKY45-GFP and 35S::GFP protein in Arabidopsis protoplasts. Scale bar $=20 \mu \mathrm{m}$.

normal condition as a control. Unlike phosphate starvation, GmWRKY45 responded faster to salt exposure, although the induced expression declined gradually after the initial burst (Figure 2C). Taken together, these results suggest that GmWRKY45 may play an important role in soybean growth and development and it is not only induced by phosphate starvation but also induced by salt stress.

\section{Overexpression of GmWRKY45 Affects the Adaptation of Transgenic Arabidopsis to Phosphate Starvation}

To functionally characterize the role of GmWRKY45 in response and adaptation to phosphate starvation, we constructed GmWRKY45-overexpressing transgenic Arabidopsis (Supplemental Figure S1). Two-week-old plants of transgenic 
A

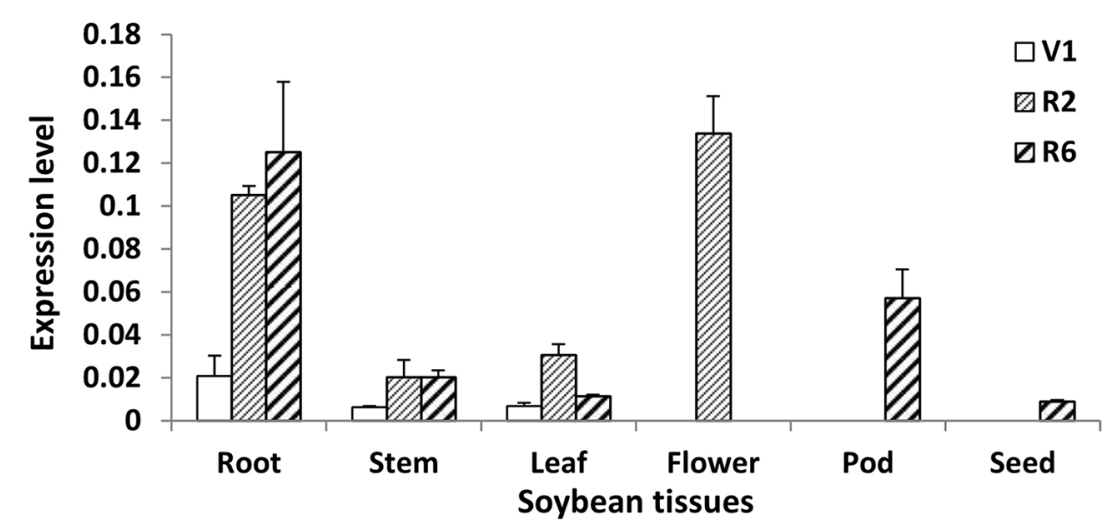

B

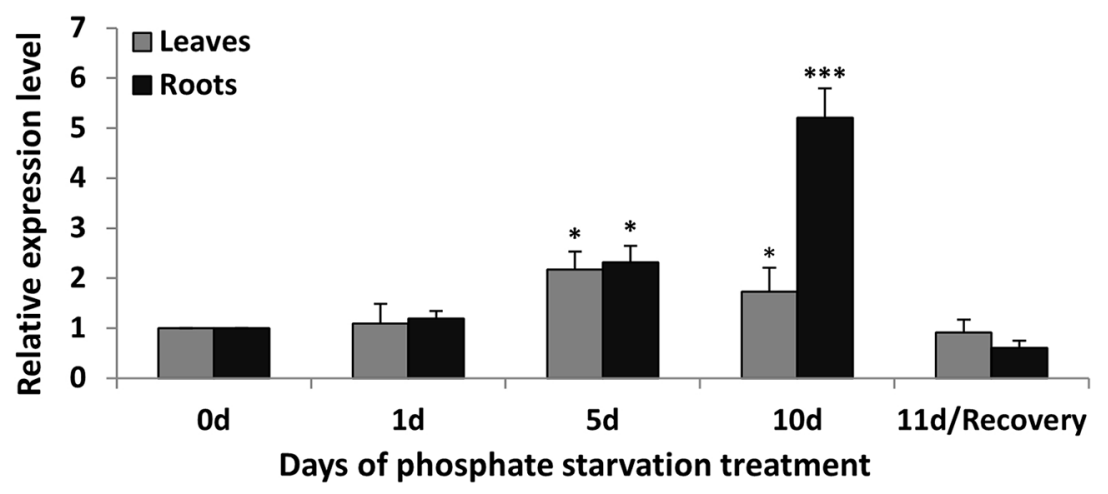

C

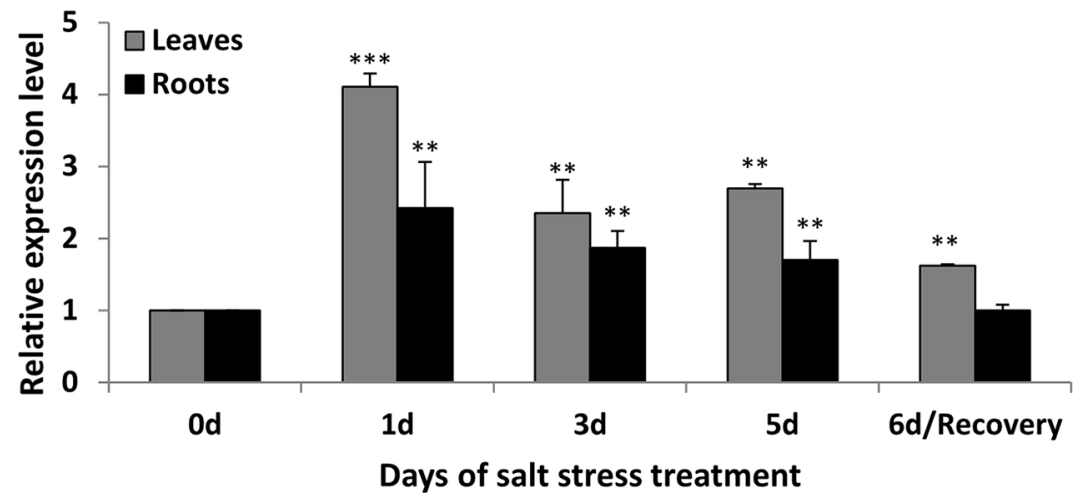

FIGURE 2 | Expression patterns of GmWRKY45 in soybean. (A) The expression of GmWRKY45 in roots, stems, leaves, flowers, pods and seeds at the first trifoliate stage (V1), full bloom stage (R2) and full seed stage (R6). (B) Time course of the expression level of GmWRKY45 in leaves and roots. Expression is relative to the transcript levels under Pi-sufficient condition (2.5 $\mathrm{MM} \mathrm{Pi}$ ). 0, 1, 5 and $10 \mathrm{~d}$, duration of Pi starvation (days); Recovery $11 \mathrm{~d}, 10$ days of Pi starvation followed by 1 day on Pi-sufficient substrate. (C) Time course of the expression level of GmWRKY45 in response to salt stress (150 Mm NaCl). 0, 1, 3, and 5 d, duration of salt stress (days); recovery $6 \mathrm{~d}, 5$ days of salt stress followed by 1 day on normal substrate. Actin was used as an internal control. Values represent mean \pm standard deviation of three biological replicates. $P$-values were calculated using Student's $t$-test and ${ }^{*} P<0.05$ and ${ }^{* *} P<0.01$ and ${ }^{* *} P<0.001$ compared with control and $\mathrm{O}$ h, respectively. 
Arabidopsis lines and the wild type Arabidopsis (WT) were exposed to a vermiculite medium containing Pi-sufficient $(\mathrm{P}+$, $1 \mathrm{mM} \mathrm{KH_{2 }} \mathrm{PO}_{4}$ ) and Pi-deficient ( $\mathrm{P}-, 0.25 \mathrm{mM} \mathrm{KH}_{2} \mathrm{PO}_{4}$ ) conditions $30 \mathrm{~d}$. Under $\mathrm{P}+$ condition, we found the growth period of all plants was basically the same, with all plants at the stage of stem elongation (Figure 3A and Supplemental Figure S2). Under the $\mathrm{P}-$ condition, the growth period of GmWRKY45-overexpressing transgenic Arabidopsis lines was not affected, while the stem elongation of WT plants was inhibited (Figure 3A and Supplemental Figure S2).

In order to further verify whether GmWRKY45 affects the adaptation of transgenic Arabidopsis to low phosphorus environment, we measured the chlorophyll and anthocyanin contents of GmWRKY45-overexpressing transgenic Arabidopsis and $\mathrm{WT}$ respectively. After $\mathrm{P}+$ conditions $30 \mathrm{~d}$, no significant differences in chlorophyll and anthocyanin contents were observed between WT and transgenic plants (Figures 3B, C). After P- condition $30 \mathrm{~d}$, significant accumulation of chlorophyll and anthocyanin was observed in the WT. However, the contents of chlorophyll and anthocyanin in the GmWRKY45overexpressing transgenic Arabidopsis lines under P-condition did not increase (Figures 3B, C). Previous studies have shown that plants produce more chlorophyll and anthocyanin under phosphorus deficiency stress (Marschner, 2011). These results mean that the low-phosphorus environment we established has a significant physiological stress effect on the WT, while the transgenic Arabidopsis is not affected. Therefore, we can determine that the overexpression of GmWRKY45 attenuates the sensitivity of Arabidopsis to phosphate starvation and improves the adaptability of Arabidopsis to phosphate starvation.

\section{GmWRKY45 Enhances Tolerance to Phosphate Starvation May Partly Relate to Changes in Root System Architecture}

Root system architecture (RSA) was one of the adaptive responses of plants to Pi status (López-Bucio et al., 2003), so it was logical to investigate GmWRKY45 effect on RSA of Arabidopsis. To test whether the better growth of transgenic Arabidopsis in phosphate starvation was related to the change of RSA, the number and length of lateral roots of WT and transgenic plants grown in vertically oriented agar plates under $\mathrm{P}+$ and $\mathrm{P}-$ conditions for 15 days were monitored (Figure 4A). In our experiment, deprivation of Pi significantly reduced lateral root growth of both WT and transgenic plants $(P \leq 0.05)$ (Figures 4A-C). However, compared with WT, both the number of lateral roots (Figure $4 \mathbf{B}$ ) and the total length (Figure 4C) of transgenic plants were significantly increased $(P \leq 0.05)$ under $\mathrm{P}-$ condition. Lateral root $(\mathrm{LR})$ plays an active role in plant phosphorus deficiency (Jia et al., 2018), so the increase of LR of transgenic Arabidopsis may be one of the reasons why transgenic plants grow faster and better than WT under $\mathrm{P}-$ condition. In addition, the LR of transgenic Arabidopsis is still more developed than WT under $\mathrm{P}+$ condition (Figures $\mathbf{4 A - C}$ ). This suggested that role of GmWRKY45 in promoting LR development may be independent of plant Pi status. In this study, we did not detect significant differences in primary root (data not shown).
Furthermore, we measured the fresh and dry weight of the plants. The GmWRKY45-overexpressing transgenic Arabidopsis exhibited better growth than WT plants, as evidenced by the greater fresh weight, dry weight both under $\mathrm{P}+$ and $\mathrm{P}$ conditions (Table 1). The inorganic phosphorus and total phosphorus concentration of the WT and transgenic Arabidopsis were also investigated. We found that the concentration of inorganic phosphorus and total phosphorus in transgenic Arabidopsis was significantly higher than WT under both $\mathrm{P}+$ and $\mathrm{P}$ - conditions (Figures 4D, E). These data proved that the regulation effect of GmWRKY45 on lateral roots not only made the transgenic Arabidopsis better adapt to Pcondition, but also performed better than the WT under $\mathrm{P}+$ condition.

\section{GmWRKY45 Regulates the Expression of Phosphate-Responsive Genes in Transgenic Arabidopsis}

In order to further clarify the mechanisms of GmWRKY45 regulating the phosphate starvation response of transgenic Arabidopsis, the expression changes of several phosphateresponsive genes were monitored by RT-qPCR. Under $\mathrm{P}+$ condition, the transcript abundance of AtSPX1, AtPHO1, AtPHT1;1, AtPHT1;4, AtPHT1;5, and AtACP5 significantly increased in transgenic Arabidopsis compared with the WT (Figure 5). The steady-state transcript levels of all the tested genes increased in WT under P-condition (Figure 5), which is consistent with previous research (Del Pozo et al., 1999; Shin et al., 2004; Wang et al., 2004; Nagarajan et al., 2011; Puga et al., 2014). However, this increase in transcription levels was not significant in transgenic Arabidopsis lines. Under P- conditions, the transcript levels of AtPHO1, AtPHT1;1, and AtACP5 did not increase in transgenic Arabidopsis, and the transcription levels of AtSPX1, AtPHT1;4, and AtPHT1;5 were observed increased, but at a reduced level than that observed in WT (Figure 5). These results indicated that GmWRKY45 enhanced the expression of these phosphate-responsive genes in transgenic Arabidopsis under normal condition, but changed the response of these phosphate-responsive genes to phosphate starvation.

\section{Overexpression of GmWRKY45 Enhances Salt Tolerance in Transgenic Arabidopsis}

Salt stress had negative effects on plant growth and development by reducing survival rate, root length and fresh weight (Yeo, 1998). Studies have shown that salt-responsive TFs deal with high concentrations of salt by maintaining high survival rates and increasing their roots' length (Hichri et al., 2017; Li et al., 2017). The results above told us that GmWRKY45 induced by salt stress (Figure 2C) and overexpression of GmWRKY45 increased the number and length of lateral roots of transgenic Arabidopsis (Figure 4C), so we speculated that overexpression of GmWRKY45 might affected the salt tolerance in transgenic Arabidopsis. To test our speculation, WT and transgenic Arabidopsis seeds were cultured on MS medium with 0 and 150 $\mathrm{mM} \mathrm{NaCl}$ for seven days. In the absence of salt stress, WT and transgenic Arabidopsis seeds showed non-significant survival rate differences (Figures 6A, B). In $150 \mathrm{mM} \mathrm{NaCl}$ medium, the survival 
A

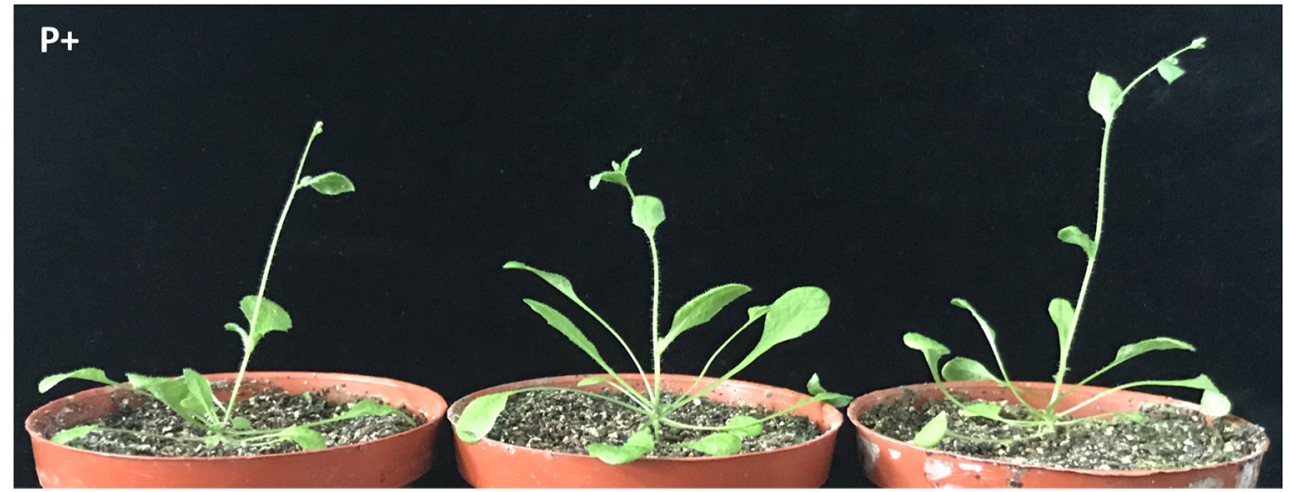

WT

OE-1

OE-4

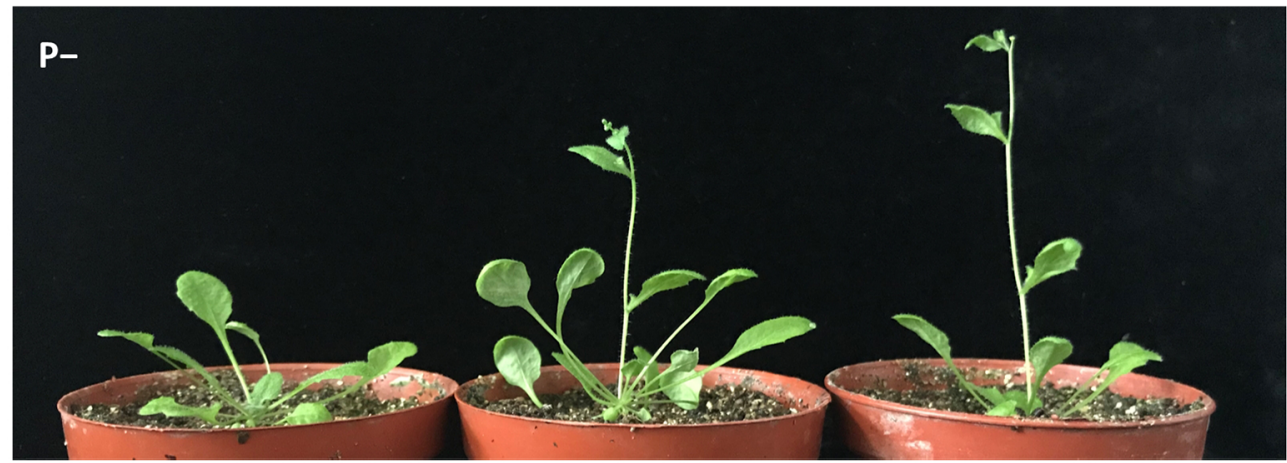

WT

OE-1

OE-4

B

C
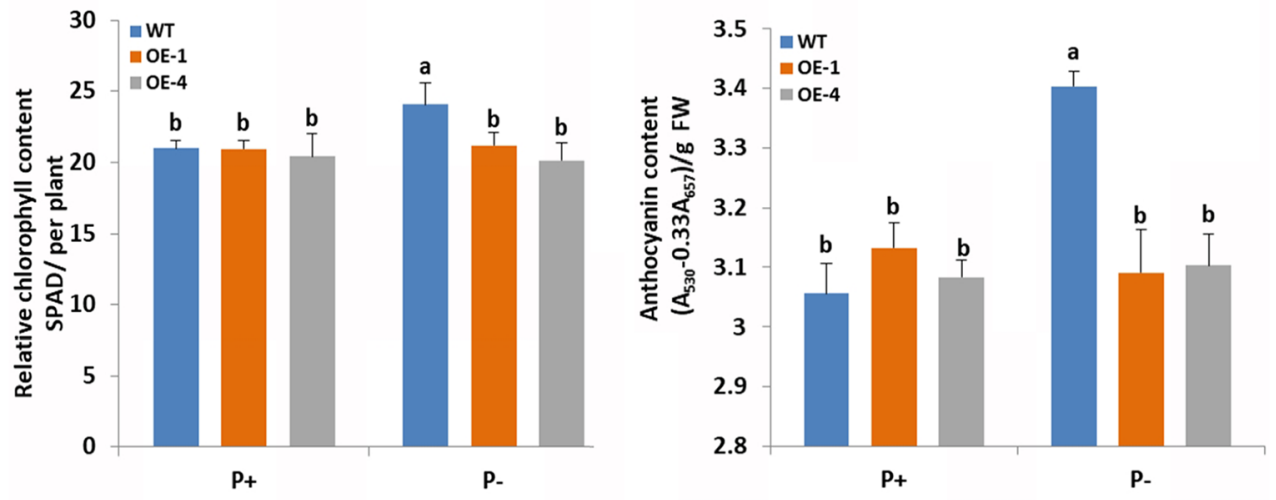

FIGURE 3 | GmWRKY45 enhances tolerance to phosphate starvation in Arabidopsis. (A) Two-week-old seedlings were grown in the greenhouse for 30 d under P+ (1 mM Pi) or P- (0.25 mM Pi) conditions. Comparison of chlorophyll (B) and anthocyanin (C) contents of WT and transgenic Arabidopsis plants (OE-1 and OE-4) in plants grown in the greenhouse for $30 \mathrm{~d}$ under $\mathrm{P}+$ and $\mathrm{P}$ - conditions. Data are means of three replicates with errors bars indicating SD. Means with different letters are significantly different (one-way ANOVA, Duncan, $P \leq 0.05$ ). The scale bars in the panels represent $1 \mathrm{~cm}$.

rate of transgenic lines was significantly higher than that of WT $(P<$ 0.001) (Figures 6A, B). The experiments were repeated for three times and the results were consistent. One set of the experiments was shown.
Plant cells with more soluble sugar accumulation had better tolerance to salt stress (Santa-Cruz et al., 1999). In order to evaluate the physiological changes of transgenic plants under salt stress, the content of soluble sugar as osmotic regulators in WT 
A
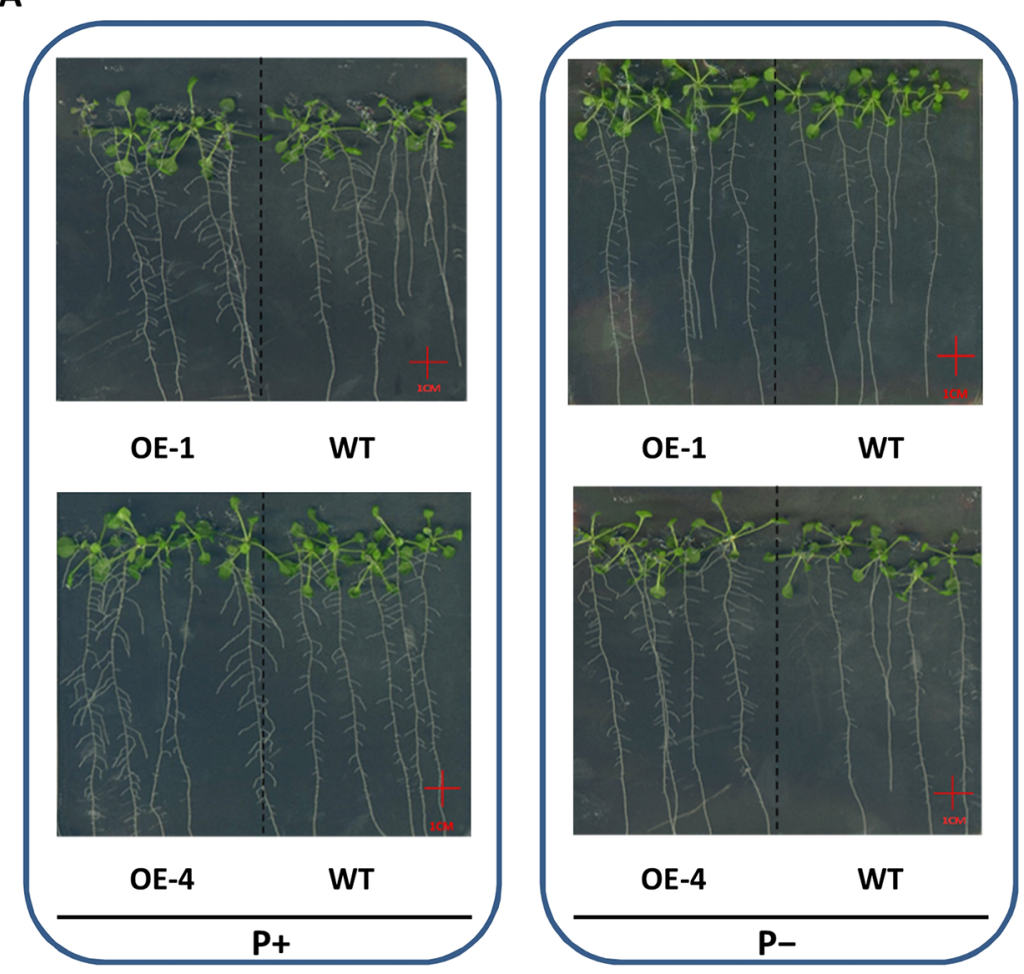

B

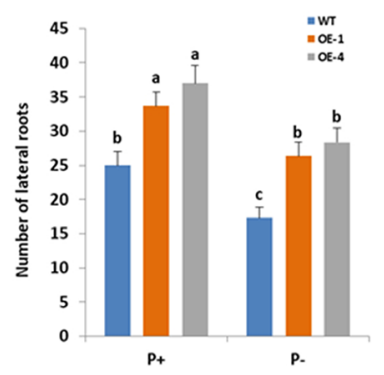

D

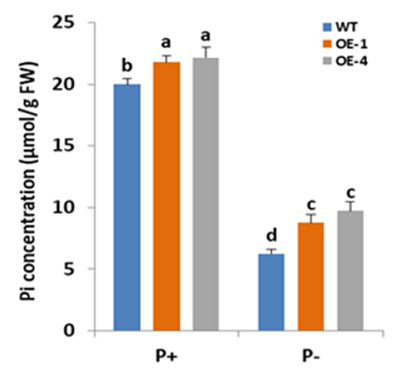

C

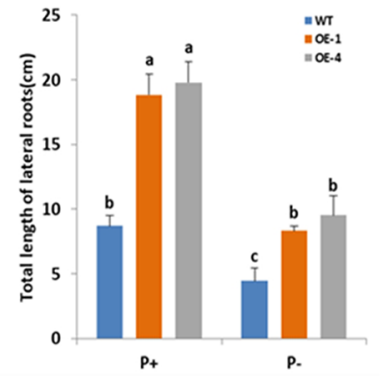

E

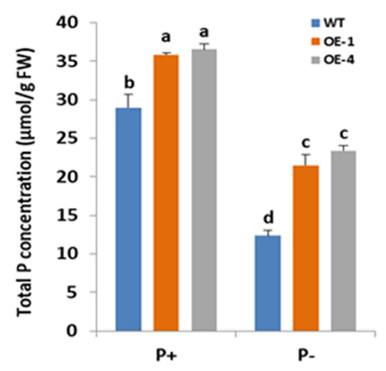

FIGURE 4 | Overexpression of GmWRKY45 increased lateral roots growth and phosphorus content in transgenic Arabidopsis. (A) WT and GmWRKY45overexpressing transgenic Arabidopsis were grown under $\mathrm{P}+$ and $\mathrm{P}$ - conditions for 15 days on vertically oriented petri plates. Total number of lateral roots per plant (B) and total length of lateral roots per plant (C) was determined in WT and transgenic Arabidopsis plants grown with P+ and P- on the 15 day. Inorganic phosphorus (D) and total phosphorus (E) contents were determined in WT and transgenic Arabidopsis plants grown with $\mathrm{P}+$ and $\mathrm{P}$ - on the 15 day. Values are mean \pm SE $(n=3)$, and different letters above the bars indicate that the means are statistically different (one-way ANOVA, Duncan, $P \leq 0.05)$. 
TABLE 1 | Comparative values of fresh and dry weight of plants at 15 days under $\mathrm{P}+$ and $\mathrm{P}$ - conditions, and the values are mean \pm SD of three independent experiments with 5 seedlings being used in each experiment $(P$-values were calculated using Student's t-test and ${ }^{\star \star *} P<0.001$ compared with WT).

\begin{tabular}{lcc}
\hline Genotype & Fresh weight $(\mathbf{m g})$ & Dry weight $(\mathbf{m g})$ \\
\hline $\mathrm{P}+(1 \mathrm{mM} \mathrm{Pi})$ & & \\
WT & $34.44 \pm 3.67$ & $2.46 \pm 0.25$ \\
OE-1 & $58.24 \pm 2.82^{\star \star \star}$ & $4.3 \pm 0.65^{\star \star \star}$ \\
OE-4 & $60.66 \pm 2.76^{\star \star \star}$ & $4.36 \pm 0.68^{\star \star \star}$ \\
P-(0.25mM Pi) & & \\
WT & $23.12 \pm 1.86$ & $1.88 \pm 0.16$ \\
OE-1 & $39.72 \pm 1.79^{\star \star \star}$ & $3.42 \pm 0.13^{\star \star \star}$ \\
OE-4 & $40.16 \pm 2.32^{\star \star \star}$ & $3.46 \pm 0.23^{\star \star \star}$
\end{tabular}
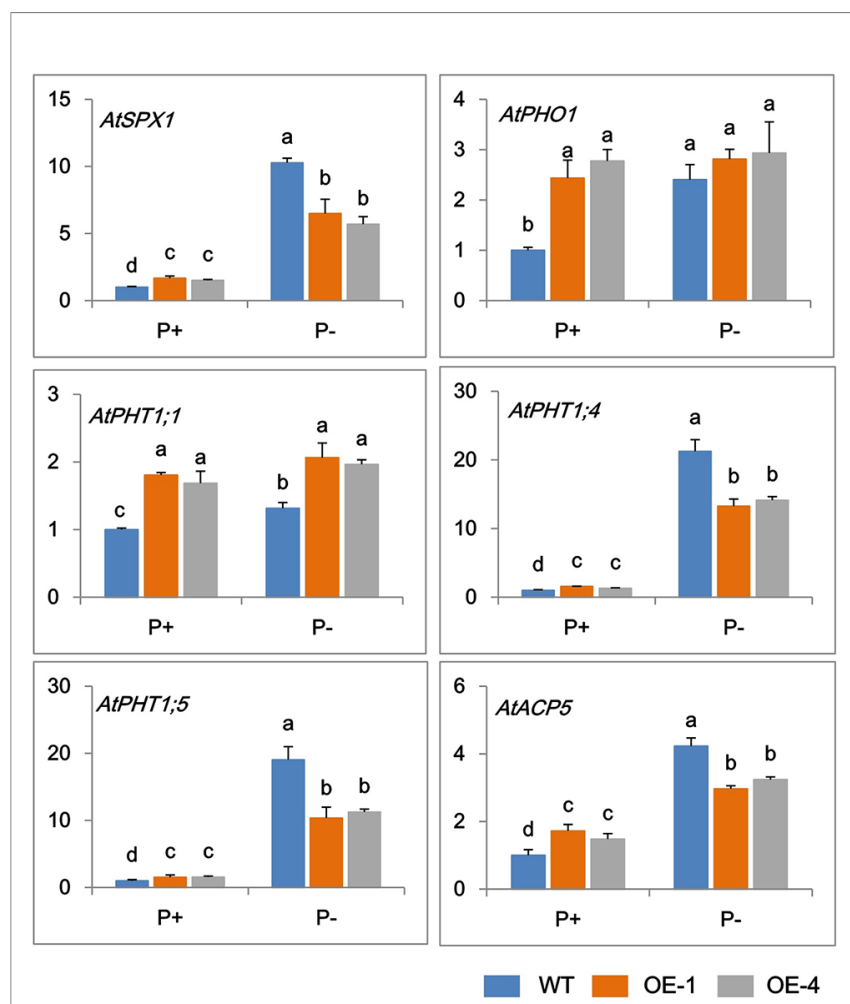

FIGURE 5 | Expression change of phosphate-responsive genes in WT and GmWRKY45-overexpressing transgenic Arabidopsis plants. Total RNA samples were extracted from plants of seedling was done after growing them for 15 days on medium with $\mathrm{P}+$ and $\mathrm{P}$-. Expression was normalized to that of Actin. Data are means $\pm S D(n=3)$. Means with different letters are significantly different (one-way ANOVA, Duncan, $P \leq 0.05$ ).

and transgenic Arabidopsis were measured. Transgenic Arabidopsis seedlings of two-week-old were treated with 150 $\mathrm{mM} \mathrm{NaCl}$ for 5 days and WT seedlings were used as the control. After salt stress, seedlings of GmWRKY45-overexpressing transgenic Arabidopsis showed similar growth compared to WT seedlings (data not shown), but the transgenic Arabidopsis showed higher levels of soluble sugars compared to the WT (Figure 7). These results indicate that GmWRKY45 may function in plant survival mechanism under high salinity levels and its salt resistance may be controlled by increasing soluble sugar in plants. Specifically, the soluble sugar content of WT and transgenic Arabidopsis both decreased rapidly on the 5th day of salt stress (Figure 7). We analyzed that it might be the continuous salt stress that caused the abnormal growth of plants.

\section{Overexpression of GmWRKY45 Changes Fertility of Transgenic Arabidopsis}

Many WRKY genes have been reported to affect plant development and growth throughout the entire development and growth period (Jiang and Yu, 2009; Zhang et al., 2010; Yang et al., 2016). In order to study the effect of GmWRKY45 on the growth and development of Arabidopsis, we tested the growth phenotypes of WT and GmWRKY45-overexpressing transgenic Arabidopsis. At the early stage of plant growth, we did not find significant differences between transgenic Arabidopsis and WT. Interestingly, we found that the silique of the transgenic Arabidopsis was significantly shorter than WT at podding stage (Figure 8A), so we speculated that the overexpression of GmWRKY45 might affect the fertility of the transgenic Arabidopsis. We selected the $1^{\text {st }}, 5^{\text {th }}, 10^{\text {th }}$, and $15^{\text {th }}$ silique from the bottom to the top, to measure the length and number of seeds per silique. At the same time, we decolorized them with alcohol and further observation under the microscope. We found that the silique of transgenic Arabidopsis was not only significantly shorter than WT, but also the number of seeds in the silique of transgenic Arabidopsis was also significantly less than WT (Figure 8B and Table 2). All transgenic lines had the same phenotype (Supplemental Figure S3). Here, we can determined that the fertility of transgenic Arabidopsis was affected. However, no differences were observed between flowers of transgenic Arabidopsis and WT (Figure 8C). Previous studies have shown that the development of male gametes in Arabidopsis requires a pollen specific transcription factor WRKY34 and its homologous gene WRKY2 (Lei et al., 2017). In view of the pollen development was closely related to plant fertility, we tried to carry out Alexander staining on the pollens of transgenic plants and WT. We found that nearly half of the pollens of transgenic plants were sterile which indicated that the fertility change of transgenic Arabidopsis could be caused by pollen abortion (Figure 8D).

In addition, we found that transgenic seeds looked larger than WT seeds in Figure 8B, so we further observed and compared the seeds size of GmWRKY45-overexpressing transgenic Arabidopsis and WT. We randomly selected transgenic lines and WT seeds for further observation under the microscope, and we found that the transgenic seeds were indeed much larger (Figure 9A). Further, we determined seed length and width, and we found that the seeds of transgenic Arabidopsis were significantly longer and wider than the WT (Figure 9B). On the other hand, we measured the total yield per plant. We found that although the fertility of transgenic plants decreased, there was no difference in total yield between transgenic lines and WT (Supplemental Figure S4). These results confirm that the seeds of transgenic Arabidopsis become larger.

\section{DISCUSSION}

WRKY transcription factors play multiple roles in regulating plant developmental processes and responses to environmental 
A
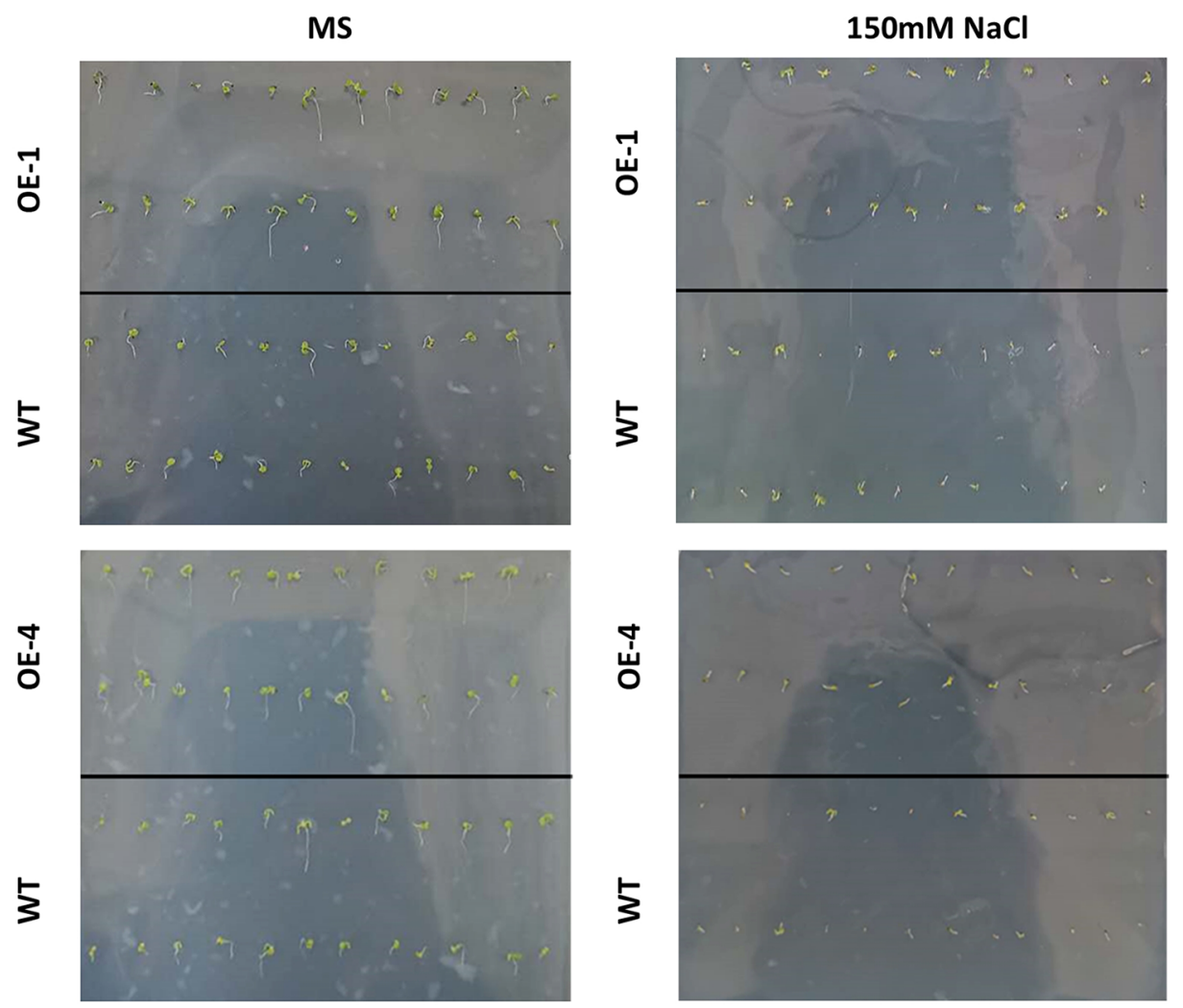

B

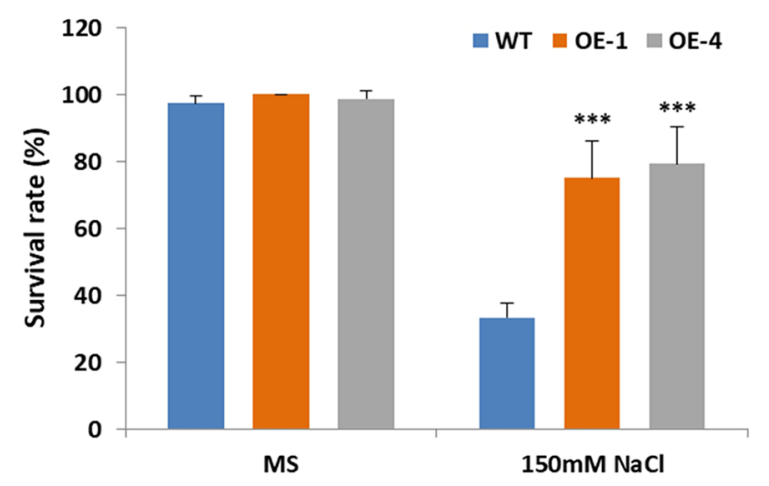

FIGURE 6 | Survival rate of Arabidopsis seeds under different NaCl concentration treatments. (A) Survival situation of WT and GmWRKY45-overexpressing transgenic Arabidopsis seeds under 0 and $150 \mathrm{mM} \mathrm{NaCl}$ treatments. Photographs were taken at the end of experiments, after seven days. (B) Survival rate differences between WT and transgenic Arabidopsis seeds under 0 and $150 \mathrm{mM} \mathrm{NaCl}$ treatments, after 7 days. $P$-values were calculated using Student's $t$-test and ${ }^{\star \star \star} P<0.001$ compared with WT. Error bars represent SE $(\mathrm{n}=3)$.

challenges (Rushton et al., 2010), so in recent years, scientists have taken a keen interest in it. However, most functional analyses of WRKY transcription factors have been restricted to A. thaliana and O. sativa, and few WRKY family members from soybean have been reported relatively. In this study, a WRKY family gene was found in soybean by homology comparison, named GmWRKY45. GmWRKY45 and AtWRKY45 share similar structures and high sequence homology according to multiple comparisons and phylogenetic relationship analysis (Figures 1A, B). In the subcellular localization test, we found that GmWRKY45 was localized in the nucleus (Figure 1C). Thus, GmWRKY45 was a member of the soybean WRKY TFs family, 


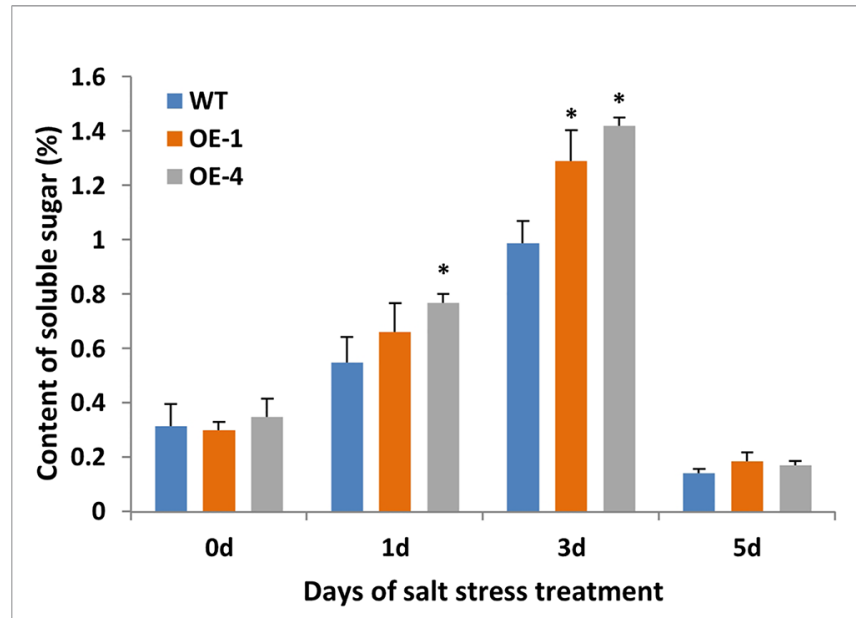

FIGURE 7 | Soluble sugar content of WT and GmWRKY45-overexpressing transgenic Arabidopsis plants. P-values were calculated using Student's $t$-test and ${ }^{\star} P<0.05$ compared with WT. Error bars represent SE $(n=3)$.

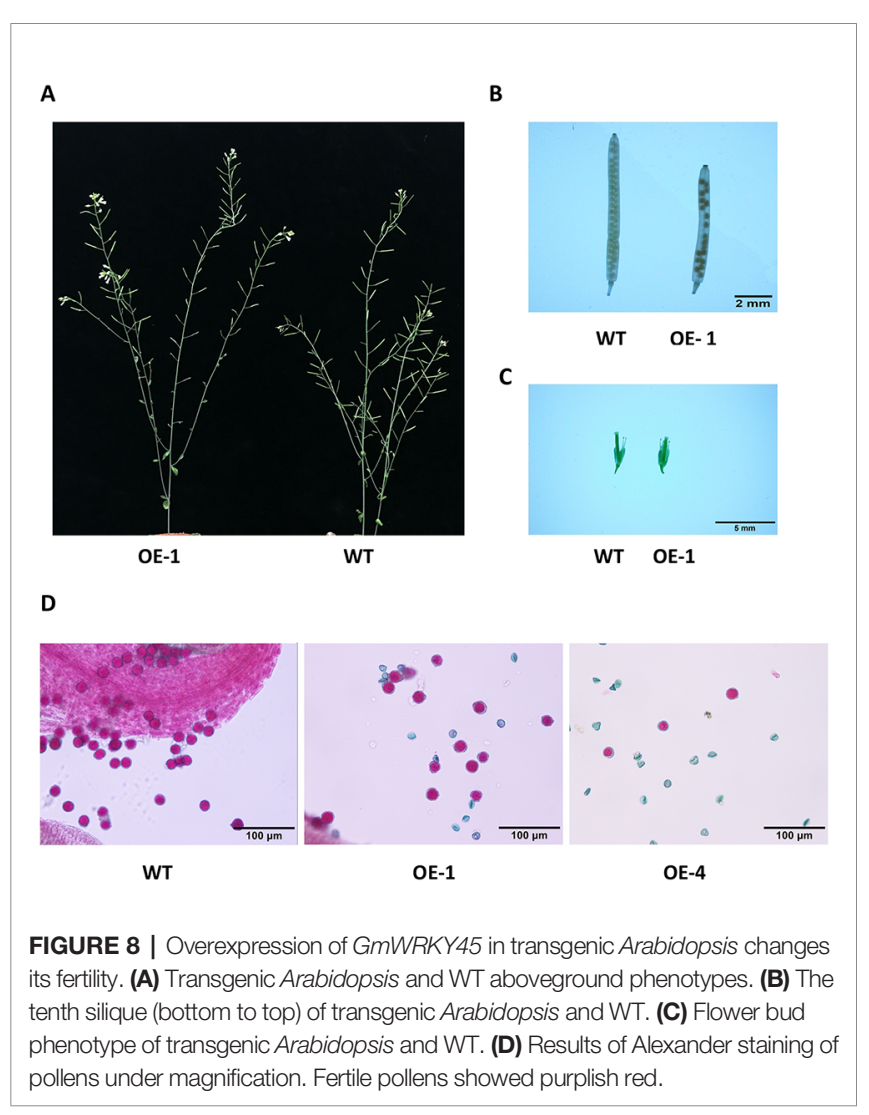

and might function in the nucleus. RT-qPCR showed that the expression of GmWRKY45 not only was induced by phosphate starvation, but was also induced by salt stress (Figures 2B, C). These facts suggest that GmWRKY45 expression in soybean is not just related to one abiotic stress, like many other WRKY transcription factors such as OsWRKY74, not only was induced by deficiency of Pi and Fe, but also inhibited by $\mathrm{N}$ deficiency and cold stress (Dai et al., 2015). GmWRKY45 was expressed in all tissues of soybean plants and strongly expressed in roots, flowers
TABLE 2 | Plant silique length and number of seeds of WT and transgenic plants at podding stage $\left(1^{\text {st }} \mathrm{S} / \mathrm{P}\right.$ was representing the first silique from the bottom to up, other and so on), the values were mean \pm SD of three independent experiments with four siliques were used in each experiment $(P$-values were calculated using Student's t-test and ${ }^{\star \star *} P<0.001$ compared with WT).

\begin{tabular}{lcc}
\hline Materials & Silique length $(\mathbf{m m})$ & Seed number (per silique) \\
\hline $\mathrm{WT}$ & & \\
$1^{\text {st }} \mathrm{S} / \mathrm{P}$ & $9.78 \pm 0.60$ & $23.5 \pm 1.73$ \\
$5^{\text {th }} \mathrm{S} / \mathrm{P}$ & $14.13 \pm 0.69$ & $45.75 \pm 2.75$ \\
$10^{\text {th }} \mathrm{S} / \mathrm{P}$ & $14.52 \pm 0.63$ & $51.5 \pm 1.29$ \\
$15^{\text {th }} \mathrm{S} / \mathrm{P}$ & $14.58 \pm 0.36$ & $55.5 \pm 2.38$ \\
$\mathrm{OE}-1$ & & \\
$1^{\text {st }} \mathrm{S} / \mathrm{P}$ & $8.92 \pm 0.50$ & $11.25 \pm 1.50^{\star \star \star}$ \\
$5^{\text {th }} \mathrm{S} / \mathrm{P}$ & $8.32 \pm 0.53^{\star \star \star}$ & $14.75 \pm 0.95^{\star \star \star}$ \\
$10^{\text {th }} \mathrm{S} / \mathrm{P}$ & $9.70 \pm 0.66^{\star \star \star}$ & $18.25 \pm 1.50^{\star \star \star}$ \\
$15^{\text {th }} \mathrm{S} / \mathrm{P}$ & $8.84 \pm 0.27^{\star \star \star}$ & $11.50 \pm 0.57^{\star \star \star}$ \\
$\mathrm{OE}-4$ & & \\
$1^{\text {st }} \mathrm{S} / \mathrm{P}$ & $8.68 \pm 0.34$ & $11.00 \pm 1.82^{\star \star \star}$ \\
$5^{\text {th }} \mathrm{S} / \mathrm{P}$ & $8.03 \pm 0.65^{\star \star \star}$ & $15.25 \pm 0.95^{\star \star \star}$ \\
$10^{\text {th }} \mathrm{S} / \mathrm{P}$ & $9.26 \pm 0.57^{\star \star \star}$ & $18.50 \pm 1.29^{\star \star \star}$ \\
$15^{\text {th }} \mathrm{S} / \mathrm{P}$ & $8.75 \pm 0.42^{\star \star \star}$ & $12.25 \pm 0.95^{\star \star \star}$ \\
\hline
\end{tabular}

and pods tissues which may hint at its function (Figure 2A). Furthermore, we built the GmWRKY45 pro::GUS transgenic Arabidopsis lines to further reveal the tissue expression pattern of GmWRKY45. Unfortunately, GmWRKY45 pro::GUS activity was not detected in GmWRKY45 promoter Arabidopsis at all growth stages (data not shown), indicating that its promoter

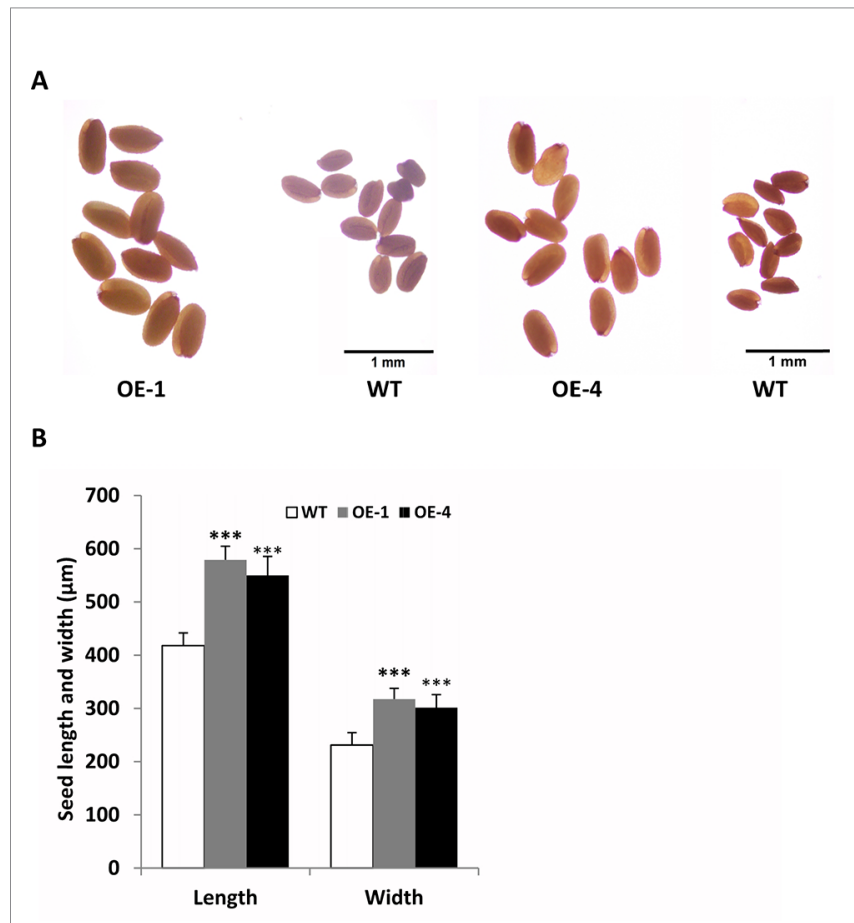

FIGURE 9 | Overexpression of GmWRKY45 in transgenic Arabidopsis changes its seed size. (A) Transgenic Arabidopsis compared with the WT on seed size comparison (B) Width and length of seeds from the transgenic Arabidopsis and WT. Data significantly different from the WT are indicated $\left({ }^{\star \star \star} P<0.001\right.$, Student's $t$-test). Error bars represent SE $(n=3)$. 
maybe species-specific or the $2-\mathrm{kb}$ region used in this experiment may not be sufficient.

In order to further study the functions of GmWRKY45 in plants, we transformed the gene into Arabidopsis due to the difficulty of soybean transformation. Arabidopsis has been used in transgenic research for stress-tolerant genes from crops that are not easy for gene transformation analysis including soybean and wheat (Hao et al., 2011; He et al., 2011). We found that overexpression of GmWRKY45 improved tolerance to phosphate starvation in transgenic Arabidopsis plants as under P-condition its growth was not inhibited relative to the WT (Figure 3A and Supplemental Figure S2). Through further physiological measurements, we found that the content of chlorophyll and anthocyanin did not increase in transgenic Arabidopsis in the low-phosphorus environment, while that in the WT increased significantly (Figures 3B, C). That's not surprising. Lack of P causes plant cells to grow slowly, so the levels of chlorophyll are relatively high. Slow cell growth is also beneficial to anthocyanin formation (Devaiah et al., 2007; Marschner, 2011). Although GmWRKY45 improved tolerance to phosphate starvation similarly as AtWRKY45 in transgenic Arabidopsis plants, they may use different approaches. AtWRKY45 is involved in Arabidopsis response to phosphate starvation by direct upregulation of AtPHT1;1 expression (Wang et al., 2014). In our study, we found the transgenic Arabidopsis has better adaptability to phosphate starvation, which may be partly due to overexpression of GmWRKY45 improving lateral root development in transgenic Arabidopsis (Figure 4 and Table 1). Lateral root initiation and elongation may play an important role in the uptake of immobile nutrients such as phosphorus by increasing soil exploration and phosphorus acquisition (Zhu et al., 2005). In addition, we found that transgenic Arabidopsis had higher survival rate (Figure 6) and content of soluble sugar (Figure 7) compared with WT under salt stress. Overexpression of GmWRKY45 results in partial abortion of the pollens, shorter silique and fewer seeds per pod in Arabidopsis (Figure 8, Table 2, and Supplemental Figure S3). However, although the number of seeds decreased, the yield of seed per plant did not decrease, which might be due to the transgenic Arabidopsis seed size larger than WT (Figure 9 and Supplemental Figure S4). Studies have shown that the expression difference of SoyWRKY15a can affect soybean seed size (Gu et al., 2017), and GmWRKY45 may be another WRKY protein related to seed size regulation in soybean. These results show that GmWRKY45 has a complex regulatory network in plants and is closely related to plant stress resistance and development.

Some phosphate-responsive genes have been shown to play an important role in phosphate response in Arabidopsis. AtSPX1 is one of Pi-dependent inhibitors of PHOSPHATE STARVATION RESPONSE 1 (PHR1) activity and is highly responsive to Pi starvation (Puga et al., 2014). AtPHO1 has previously been shown to participate in the transport of Pi from roots to shoots, and the gene expression profile normally triggered by $\mathrm{Pi}$ deficiency is suppressed in plants with low AtPHO1 expression (Rouached et al., 2011). AtPHT1;1, AtPHT1;4, and AtPHT1;5 belong to members of the
Arabidopsis Pht1 phosphate transporter family. AtPHT1;1 and AtPHT1;4 play a major role in phosphate acquisition from both low- and high-phosphate environments (Shin et al., 2004), AtPHT1;5 plays a critical role in mobilizing Pi from $\mathrm{P}$ source to sink organs in accordance with developmental cues and $\mathrm{P}$ status (Nagarajan et al., 2011). AtACP5 is a type 5 acid phosphatase gene from Arabidopsis which is induced by phosphate starvation and other types of phosphate mobilising/ oxidative stress conditions (Del Pozo et al., 1999). Our results showed the GmWRKY45-overexpressing transgenic Arabidopsis enhanced the expression of these phosphate-responsive genes under Pi-sufficient condition (Figure 5), which might be one of the reasons why transgenic Arabidopsis had highter $\mathrm{P}$ concentration than WT (Figures 4D, E). Under Pi-deficient condition, the expression of these phosphate-responsive genes was increased in GmWRKY45-overexpressing transgenic Arabidopsis, but the increase was not stronger than WT (Figure 5). This may mean that overexpression of GmWRKY45 reduces the sensitivity of Arabidopsis to low phosphorus environment. The expression of several phosphateresponsive genes was reduced in low phosphorus insensitive mutants grown in low P media (Lenin et al., 2006).

Lateral root (LR) development plays an important role in many plant stress tolerances, not only in phosphate starvation. For example, excessive $\mathrm{Fe}$ has different effects on the development of LR in different parts of Arabidopsis root system, and the inhibition of LR activation initiation is only seen in roots newly form during excess Fe exposure (Kronzucker et al., 2015); Salt stresses causes an extended quiescent phase in postemergence LRs whereby the rate of growth is suppressed for several days and that is correlated with sustaining ABA response in LRs (Lina et al., 2013); Poplar PtabZIP1-like enhances LR formation and biomass growth under drought stress (Dash et al., 2017). In our study, we found that the lateral roots of GmWRKY45-overexpressing transgenic Arabidopsis to grow well even under stress-free condition (Figure 4), which means that transgenic Arabidopsis may also perform well in other adverse soil conditions. We used PlantCARE (http:// bioinformatics.psb.ugent.be/webtools/plantcare/html/) to analyze the promoter of GmWRKY45 and found that contains many environmental stress responses and plant hormone response cis elements (Supplemental Table S5). In this paper, only phosphate starvation and salt stress are examined, so further studies are needed to clarify GmWRKY45 expression and the performance of the transgenic Arabidopsis plants in other adverse soil conditions. Furthermore, improved root systems and survival rate are considerable interest in agriculture; the pollen fertility and seed size profoundly influence total harvest. Thus, it is meaningful to study the underlying regulatory molecular mechanisms in greater detail.

\section{CONCLUSIONS}

In summary, this study characterized GmWRKY45, a WRKY TFs belonging to group IIc of the WRKY protein family that is 
localized at the nucleus in soybean. The GmWRKY45 protein acts as an important regulator of phosphate starvation responses, so that overexpression of GmWRKY45 resulted in enhanced tolerance to phosphate starvation, more and longer lateral roots, and changed expression of phosphate-responsive genes in transgenic Arabidopsis plants. In addition, our results also showed that GmWRKY45 enhanced tolerance to salt stress, and changed fertility in transgenic Arabidopsis. Further works on the role of GmWRKY45 to other abiotic stress are under way in our laboratory. We are also working on the construction of soybean overexpression and mutant materials, with a view to further studying the regulatory mechanism of GmWRKY45 on abiotic stress and development of soybean.

\section{DATA AVAILABILITY STATEMENT}

Publicly available datasets were analyzed in this study. This data can be found here: Glyma.03g220800.

\section{REFERENCES}

Abel, S., and Theologis, A. (1994). Transient transformation of arabidopsis leaf protoplasts: a versatile experimental system to study gene expression. Plant J. 5 (3), 421-427. doi: 10.1111/j.1365-313X.1994.00421.x

Abràmoff, M. D., Magalhães, P. J., and Ram, S. J. (2004). Image processing with ImageJ. Biophotonics Int. 11 (7), 36-42. doi: 10.1002/9780470612491.ch8

Baek, D., Kim, M. C., Chun, H. J., Kang, S., Park, H. C., Shin, G., et al. (2013). Regulation of miR399f Transcription by AtMYB2 Affects Phosphate Starvation Responses in Arabidopsis. Plant Physiol. 161 (1), 362-373. doi: 10.1104/ pp.112.205922

Bencke-Malato, M., Cabreira, C., Wiebke-Strohm, B., Bücker-Neto, L., Mancini, E., Osorio, M. B., et al. (2014). Genome-wide annotation of the soybean WRKY family and functional characterization of genes involved in response to Phakopsora pachyrhizi infection. BMC Plant Biol. 14 (1), 236. doi: 10.1186/s12870-014-0236-0

Chen, F., Hu, Y., Vannozzi, A., Wu, K., Cai, H., Qin, Y., et al. (2017). The WRKY transcription factor family in model plants and crops. Crit. Rev. In Plant Sci. 36 (5-6), 311-335. doi: 10.1080/07352689.2018.1441103

Chou, K.-C., and Shen, H.-B. (2008). Cell-PLoc: a package of Web servers for predicting subcellular localization of proteins in various organisms. Nat. Protoc. 3 (2), 153. doi: 10.1038/nprot.2007.494

Clough, S. J., and Bent, A. F. (1998). Floral dip: a simplified method for agrobacterium-mediated transformation of arabidopsis thaliana. Plant J. 16 (6), 735-743. doi: 10.1046/j,1365-313x.1998.00343.x

Dai, X., Wang, Y., and Zhang, W.-H. (2015). OsWRKY74, a WRKY transcription factor, modulates tolerance to phosphate starvation in rice. J. Exp. Bot. 67 (3), 947-960. doi: 10.1093/jxb/erv515

Dash, M., Yordanov, Y. S., Georgieva, T., Tschaplinski, T. J., Yordanova, E., and Busov, V. (2017). Poplar PtabZIP1-like enhances lateral root formation and biomass growth under drought stress. Plant J. 89 (4), 692. doi: 10.1111/tpj.13413

Del Pozo, J. C., Allona, I., Rubio, V., Leyva, A., De La Peña, A., Aragoncillo, C., et al. (1999). A type 5 acid phosphatase gene from Arabidopsis thaliana is induced by phosphate starvation and by some other types of phosphate mobilising/oxidative stress conditions. Plant J. 19 (5), 579-589. doi: 10.1046/ j.1365-313X.1999.00562.x

Devaiah, B. N., Karthikeyan, A. S., and Raghothama, K. G. (2007). WRKY75 transcription factor is a modulator of phosphate acquisition and root development in Arabidopsis. Plant Physiol. 143 (4), 1789-1801. doi: 10.2307/ 40065391

\section{AUTHOR CONTRIBUTIONS}

CL and SY designed the experiments. CL, XL, HR, JZ, and FX finished the experiments. CL and XL analyzed the data. CL finished the manuscript. SY and JG revised the paper. All authors approved the paper.

\section{FUNDING}

This work was supported by the National Transgene Science and Technology Major Program of China (2016ZX08004-005), the Fundamental Research Funds for the Central Universities (KYT201801) and the Program for Changjiang Scholars and Innovative Research Team in University (PCSIRT_17R55).

\section{SUPPLEMENTARY MATERIAL}

The Supplementary Material for this article can be found online at: https://www.frontiersin.org/articles/10.3389/fpls.2019. 01714/full\#supplementary-material

Eulgem, T., and Somssich, I. E. (2007). Networks of WRKY transcription factors in defense signaling. Curr.Opin.Plant Biol. 10 (4), 366-371. doi: 10.1016/ j.pbi.2007.04.020

Eulgem, T., Rushton, P. J., Robatzek, S., and Somssich, I. E. (2000). The WRKY superfamily of plant transcription factors. Trends In Plant Sci. 5 (5), 199-206. doi: 10.1016/S1360-1385(00)01600-9

Gu, Y., Li, W., Jiang, H., Wang, Y., Gao, H., Liu, M., et al. (2017). Differential expression of a WRKY gene between wild and cultivated soybeans correlates to seed size. J. Exp. Bot. 68 (11), 2717-2729. doi: 10.1093/jxb/erx147

Hao, Y. J., Wei, W., Song, Q. X., Chen, H. W., Zhang, Y. Q., Wang, F., et al. (2011). Soybean NAC transcription factors promote abiotic stress tolerance and lateral root formation in transgenic plants. Plant J. 68 (2), 302-313. doi: 10.1111/ j.1365-313x.2011.04687.x

He, X., Hou, X., Shen, Y., and Huang, Z. (2011). TaSRG, a wheat transcription factor, significantly affects salt tolerance in transgenic rice and Arabidopsis. FEBS Lett. 585 (8), 1231-1237. doi: 10.1016/j.febslet.2011.03.055

Hichri, I., Muhovski, Y., Žižková, E., Dobrev, P. I., Gharbi, E., Franco-Zorrilla, J. M., et al. (2017). The Solanum lycopersicum WRKY3 transcription factor SIWRKY3 is involved in salt stress tolerance in tomato. Front. In Plant Sci. 8, 1343. doi: 10.3389/fpls.2017.01343

Jia, X., Liu, P., and Lynch, J. P. (2018). Greater lateral root branching density in maize improves phosphorus acquisition from low phosphorus soil. J. Exp. Bot. 69 (20), 4961-4970. doi: 10.1093/jxb/ery252

Jiang, W., and Yu, D. (2009). Arabidopsis WRKY2 transcription factor mediates seed germination and postgermination arrest of development by abscisic acid. BMC Plant Biol. 9 (1), 96. doi: 10.1186/1471-2229-9-96

Krueger, K., Goggi, A. S., Mallarino, A. P., and Mullen, R. E. (2013). Phosphorus and potassium fertilization effects on soybean seed quality and composition. Crop Sci. 53 (2), 602-. doi: 10.2135/cropsci2012.06.0372

Kim, J., Yi, H., Choi, G., Shin, B., Song, P.-S., and Choi, G. (2003). Functional characterization of phytochrome interacting factor 3 in phytochromemediated light signal transduction. Plant Cell 15 (10), 2399-2407. doi: 10.1105/tpc.014498

Kronzucker, H. J., Li, G., Li, B., Song, H., and Shi, W. (2015). AUX1 and PIN2 Protect Lateral Root Formation in Arabidopsis under Fe Stress. Plant Physiol. 169 (4), pp.00904.02015. doi: 10.1104/pp.15.00904

López-Bucio, J., Cruz-Ramırez, A., and Herrera-Estrella, L. (2003). The role of nutrient availability in regulating root architecture. Curr. Opin. In Plant Biol. 6 (3), 280-287. doi: 10.1016/S1369-5266(03)00035-9 
Lei, R., Li, X., Ma, Z., Lv, Y., Hu, Y., and Yu, D. (2017). Arabidopsis WRKY 2 and WRKY 34 transcription factors interact with VQ 20 protein to modulate pollen development and function. Plant J. 91 (6), 962-976. doi: 10.1111/tpj.13619

Lenin, S. C., José, L. B., Alejandra, C. L., Abel, G. O., Esmeralda, H. A., and Luis, H. E. (2006). Characterization of low phosphorus insensitive mutants reveals a crosstalk between low phosphorus-induced determinate root development and the activation of genes involved in the adaptation of Arabidopsis to phosphorus deficiency. Plant Physiol. 140 (3), 879-889. doi: 10.2307/20205654

Li, Y., Chen, Q., Nan, H., Li, X., Lu, S., Zhao, X., et al. (2017). Overexpression of GmFDL19 enhances tolerance to drought and salt stresses in soybean. PloS One 12 (6), e0179554. doi: 10.1371/journal.pone.0179554

Lina, D., Daniela, D., Han, N. C., Chan, P. M. Y., Rishikesh, B., Bennett, M. J., et al. (2013). Endodermal ABA signaling promotes lateral root quiescence during salt stress in Arabidopsis seedlings. Plant Cell 25 (1), 324-341. doi: 10.1105/ tpc.112.107227

Marschner, H. (2011). Marschner's mineral nutrition of higher plants (London: Academic Press).

Munns, R., and Tester, M. (2008). Mechanisms Salinity Tolerance. Annu. Rev. Plant Biol. 59 (1), 651-681. doi: 10.1146/annurev.arplant.59.032607.092911

Nagarajan, V. K., Ajay, J., Poling, M. D., Lewis, A. J., Raghothama, K. G., and Smith, A. P. (2011). Arabidopsis Pht1;5 mobilizes phosphate between source and sink organs and influences the interaction between phosphate homeostasis and ethylene signaling. Plant Physiol. 156 (3), 1149-1163. doi: 10.1104/ pp.111.174805

Pandey, S. P., and Somssich, I. E. (2009). The Role of WRKY Transcription Factors in Plant Immunity. Plant Physiol. 150 (4), 1648-1655. doi: 10.1104/ pp.109.138990

Phukan, U. J., Jeena, G. S., and Shukla, R. K. (2016). WRKY Transcription Factors: Molecular Regulation and Stress Responses in Plants. Front. In Plant Sci. 7 (807560), 760. doi: 10.3389/fpls.2016.00760

Puga, M. I., Mateos, I., Charukesi, R., Wang, Z., Franco-Zorrilla, J. M., de Lorenzo, L., et al. (2014). SPX1 is a phosphate-dependent inhibitor of Phosphate Starvation Response 1 in Arabidopsis. Proc. Natl. Acad. Sci. 111 (41), 14947-14952. doi: 10.1073/pnas.1404654111

Rengasamy, P. (2006). World salinization with emphasis on Australia. J. Exp. Bot. 57 (5), 1017-1023. doi: 10.1093/jxb/erj108

Rouached, H., Stefanovic, A., Secco, D., Bulak Arpat, A., Gout, E., Bligny, R., et al. (2011). Uncoupling phosphate deficiency from its major effects on growth and transcriptome via PHO1 expression in Arabidopsis. Plant J. 65 (4), 557-570. doi: 10.1111/j.1365-313X.2010.04442.x

Rushton, P. J., Somssich, I. E., Ringler, P., and Shen, Q. J. (2010). WRKY transcription factors. Trends In Plant Sci. 15 (5), 247-258. doi: 10.1016/j.tplants.2010.02.006

Santa-Cruz, A., Acosta, M., Rus, A., and Bolarin, M. C. (1999). Short-term salt tolerance mechanisms in differentially salt tolerant tomato species. Plant Physiol. Biochem. 37 (1), 65-71. doi: 10.1016/s0981-9428(99)80068-0

Scarpeci, T. E., Zanor, M. I., Carrillo, N., Mueller-Roeber, B., and Valle, E. M. (2008). Generation of superoxide anion in chloroplasts of Arabidopsis thaliana during active photosynthesis: a focus on rapidly induced genes. Plant Mol. Biol. 66 (4), 361-378. doi: 10.1007/s11103-007-9274-4

Shin, H., Shin, H. S., Dewbre, G. R., and Harrison, M. J. (2004). Phosphate transport in Arabidopsis: Pht1; 1 and Pht1; 4 play a major role in phosphate acquisition from both low-and high-phosphate environments. Plant J. 39 (4), 629-642. doi: 10.1111/j.1365-313X.2004.02161.x

Vance, C. P., Uhde-Stone, C., and Allan, D. L. (2003). Phosphorus acquisition and use: critical adaptations by plants for securing a nonrenewable resource. New Phytol. 157 (3), 423-447. doi: 10.1046/j.1469-8137.2003.00695.x
Wang, Y., Ribot, C., Rezzonico, E., and Poirier, Y. (2004). Structure and expression profile of the Arabidopsis PHO1 gene family indicates a broad role in inorganic phosphate homeostasis. Plant Physiol. 135 (1), 400-411. doi: 10.1104/ pp.103.037945

Wang, H., Xu, Q., Kong, Y.-H., Chen, Y., Duan, J.-Y., Wu, W.-H., et al. (2014). Arabidopsis WRKY45 transcription factor activates PHOSPHATE TRANSPORTER1; 1 expression in response to phosphate starvation. Plant Physiol. 164 (4), 2020-2029. doi: 10.1104/pp.113.235077

Xiang, Y., Huang, Y., and Xiong, L. (2007). Characterization of stress-responsive CIPK genes in rice for stress tolerance improvement. Plant Physiol. 144 (3), 1416-1428. doi: 10.1104/pp.107.101295

Xu, Z., Raza, Q., Xu, L., He, X., Huang, Y., Yi, J., et al. (2018). GmWRKY49, a saltresponsive nuclear protein, improved root length and governed better salinity tolerance in transgenic Arabidopsis. Front. Plant Sci. 9, 809. doi: 10.3389/ fpls.2018.00809

Yang, Y., Chi, Y., Wang, Z., Zhou, Y., Fan, B., and Chen, Z. (2016). Functional analysis of structurally related soybean GmWRKY58 and GmWRKY76 in plant growth and development. J. Exp. Bot. 67 (15), 4727-4742. doi: 10.1093/ jxb/erw252

Yeo, A. (1998). Molecular biology of salt tolerance in the context of whole-plant physiology. J. Exp. Bot. 49 (323), 915-929. doi: 10.1093/jxb/49.323.915

Yoo, S.-D., Cho, Y.-H., and Sheen, J. (2007). Arabidopsis mesophyll protoplasts: a versatile cell system for transient gene expression analysis. Nat. Protoc. 2 (7), 1565. doi: 10.1038/nprot.2007.199

Zhang, G., Chen, M., Li, L., Xu, Z., Chen, X., Guo, J., et al. (2009). Overexpression of the soybean GmERF3 gene, an AP2/ERF type transcription factor for increased tolerances to salt, drought, and diseases in transgenic tobacco. J. Exp. Bot. 60 (13), 3781-3796. doi: 10.1093/jxb/erp214

Zhang, H., Jin, J., Tang, L., Zhao, Y., Gu, X., Gao, G., et al. (2010). PlantTFDB 2.0: update and improvement of the comprehensive plant transcription factor database. Nucleic Acids Res. 39 (suppl_1), D1114-D1117. doi: 10.1093/nar/ gkq1141

Zhang, J., Zhou, X., Xu, Y., Yao, M., Xie, F., Gai, J., et al. (2016). Soybean SPX1 is an important component of the response to phosphate deficiency for phosphorus homeostasis. Plant Sci. 248, 82-91. doi: 10.1016/ j.plantsci.2016.04.010

Zhou, Q.-Y., Tian, A.-G., Zou, H.-F., Xie, Z.-M., Lei, G., Huang, J., et al. (2008). Soybean WRKY-type transcription factor genes, GmWRKY13, GmWRKY21, and GmWRKY54, confer differential tolerance to abiotic stresses in transgenic Arabidopsis plants. Plant Biotechnol. J. 6 (5), 486-503. doi: 10.1111/j.14677652.2008.00336.x

Zhu, J., Kaeppler, S. M., and Lynch, J. P. (2005). Mapping of QTLs for lateral root branching and length in maize (Zea mays L.) under differential phosphorus supply. Theor. Appl. Genet. 111 (4), 688-695. doi: 10.1007/s00122-005-2051-3

Conflict of Interest: The authors declare that the research was conducted in the absence of any commercial or financial relationships that could be construed as a potential conflict of interest.

Copyright (c) 2020 Li, Liu, Ruan, Zhang, Xie, Gai and Yang. This is an open-access article distributed under the terms of the Creative Commons Attribution License (CC BY). The use, distribution or reproduction in other forums is permitted, provided the original author(s) and the copyright owner(s) are credited and that the original publication in this journal is cited, in accordance with accepted academic practice. No use, distribution or reproduction is permitted which does not comply with these terms. 